\title{
Vesicular Release Dynamics are Altered by Interaction between Chemical Cargo and Vesicle Membrane Lipids
}

\author{
Farzaneh Asadpour, ${ }^{\ddagger a, b}$ Xinwei Zhang, ${ }^{\ddagger a}$ Mohammad Mazloum-Ardakani, ${ }^{\text {bb }}$ Meysam Mirzaei, ${ }^{c}$ \\ Soodabeh Majdib, a Andrew G. Ewing*a
}

\begin{abstract}
The release of cargo from soft vesicles, an essential process for chemical delivery, is mediated by multiple factors. Among them, interactions between the chemical cargo species and the vesicular membrane, widely existing in all vesicles, has not been investigated to date. Yet, these interactions hold the potential to complicate the release process. We used liposomes loaded with different monoamines, dopamine (DA) and serotonin (5-HT), to simulate vesicular release and to monitor the dynamics of chemical release from isolated vesicles during vesicle impact electrochemical cytometry (VIEC). The release of DA from liposomes presents a longer release time compared to 5-HT. Modelling the release time showed that DA filled vesicles had a higher percentage of events where the time for the peak fall was better fit to a double exponential (DblExp) decay function, suggesting multiple kinetic steps in the release. By fitting to a desorption-release model, where the transmitters adsorbed to the vesicle membrane, the dissociation rates of DA and 5-HT from liposome membrane were estimated. DA has a lower desorption rate constant, which leads to slower DA release than that observed for 5-HT, whereas there is little difference in pore size. The alteration of vesicular release dynamics due to the interaction between chemical cargo and vesicle membrane lipids provides an important mechanism to regulate vesicular release in chemical and physiological processes. It is highly possible that this introduces a fundamental chemical regulation difference between transmitters during exocytosis.
\end{abstract}

\section{Introduction}

The release of cargo from soft vesicles is an essential step for chemical delivery related to various processes, including liposome drug delivery, exosome-mediated cell signaling, vesicular transport, intracellular vesicular trafficking, exocytosis, etc. Among these, exocytosis, with a key role in cell communication and possibly a therapeutic target for disease of the nervous or endocrine system, has attracted increasing attention even though its mechanism and dynamics has been investigated for decades. ${ }^{1-6}$ Electrochemical techniques, especially amperometry in combination with ultramicroelectrodes, have been developed as an approach to monitor the release of chemical cargo during the exocytotic event with high sensitivity and spatial-temporal resolution. ${ }^{7-12}$ The experimental signals of monitoring exocytosis, in the form of amperometric "spikes," usually contain complex information about vesicle geometry, cargo quantity, and release dynamics through the exocytotic pore driven and controlled by the SNARE protein complex, actin, and dynamin. ${ }^{13,14}$ For those vesicles isolated from cells, we can also trigger their cargo release (simulate exocytosis) and record the release signal again as "spikes" with our previously reported approach, called vesicle impact electrochemical cytometry (VIEC). ${ }^{15,16}$ However, some difficulties still exist in the dynamic analysis of these spike signals, especially for those involving complex release processes which are difficult to quantitatively describe.

Usually, the exocytotic spike is observed as an asymmetrical peak, different in the rising and falling time. The spike decay lasts a longer time and for some spikes this current decay can be fit to a single exponential $\left(I=\mathrm{Ae}^{-\alpha t}\right)$, whereas for other spikes to a double exponential $\left(I=\mathrm{A}_{1} \mathrm{e}^{-\alpha t}+\mathrm{A}_{2} \mathrm{e}^{-\beta t}\right)$ function. ${ }^{17,18}$ The single exponential (SigExp) decay can be easily explained with a diffusion model where the release pore is static. ${ }^{19-22}$ In contrast, the widely observed double exponential (DblExp) decay events during exocytotic release appear to indicate that additional processes besides simple diffusion are involved. Trouillon et al. ${ }^{19,20}$ and Yue et al. ${ }^{23}$ suggested that the DbIExp mode of vesicular release might result from the exocytotic pore closing mediated by membrane proteins. Oleinick et al. showed that

\footnotetext{
a. Department of Chemistry and Molecular Biology, University of Gothenburg, 41296 Gothenburg, Sweden.*E-mail:andrew.ewing@chem.gu.se

b. Department of Chemistry, Faculty of Science, Yazd University, Yazd, 89195-741, Iran.

*E-mail:mazloum@yazd.ac.ir

c. Department of Materials Science and Engineering, School of Engineering, Shiraz University, Shiraz, Iran.

†Electronic Supplementary Information (ESI) available: Detailed experimental procedures and materials; Fig. S1 and Table S1. See DOI: 10.1039/x0xx00000x

${ }^{\ddagger}$ These authors contributed equally
} 
the interaction of catecholamines with the protein dense core matrix in vesicles can lead to spikes with DblExp in the latter part of the spike. ${ }^{18}$ Interaction between chemical cargo and vesicle dense core is certainly important, ${ }^{24}$ however, interactions have been shown between the chemical cargo and membranes ${ }^{25-27}$ and these might play a broader role in vesicle release dynamics. These interactions could be broadly applicable as they will be observed in all vesicles, not only in the vesicles with a dense core. To the best of our knowledge this has not been investigated to date.

Interaction between the chemical cargo and the membrane, i.e. an adsorption-desorption process, adds an element of complication to the release process model and provide a mechanism of biological regulation. Numerical approaches, represented by the finite element simulation, provide a powerful tool for solving the problems related to complex processes which are difficult with direct analytical approaches. Based on the numerical analysis of the spike signals recorded through the VIEC technique, we can reconstruct the physical model of the entire vesicle release process ${ }^{28}$ and include an inverse estimation of the relative specific physical parameters, ${ }^{29,30}$ such as the vesicular pore size, diffusion coefficient of cargo chemicals, desorption rate constant, etc. These parameters can clearly describe the dissociation of cargo from the membrane and how it alters general vesicle release dynamics.

In this paper, we used similarly sized liposomes but containing different monoamines, dopamine (DA) and serotonin (5-HT) to simulate vesicular release. The use of liposomes avoids the influence of the vesicular dense core. ${ }^{31-34}$ We observed statistically different dynamics of release for the different monoamines. A finite element method, combined with Monte Carlo optimization, was adopted to estimate the dynamic parameters for release of monoamines from the liposomes during VIEC. We provide evidence that these amines differentially adsorb to the membrane lipids in the liposomes or vesicles changing the dynamics by which they exit the vesicle. By fitting to a desorption model, we calculated the desorption rate constants of these two monoamines from the vesicular membrane lipids. DA has a lower desorption rate constant from the liposome membrane, which leads to slower DA release than that observed for $5-\mathrm{HT}$, while there is little difference in pore size. Although this work describes the desorption-release process for transmitters exiting vesicles during VIEC, the fundamental aspects can be applied to many aspects of chemical or biological vesicle transport. The results experimentally indicate that the process of vesicular release is complicated by interaction between the chemical cargo and the vesicular membrane, thereby altering exocytotic release dynamics. ${ }^{25,26,35,36}$

\section{Results and Discussion}

Preparation and release dynamics for amine-loaded liposomes

In order to study the interaction between different neurotransmitters and the vesicle membrane, we chose to study dopamine-loaded vesicles (DLL) and serotonin-loaded vesicles (SLL). These are both transmitters that are widely distributed in the nervous systems of mammals. These transmitters were loaded into liposomes of similar size as a model of biological vesicles without proteins and cargo with different membrane adsorption properties. The preparation process followed our previous work. ${ }^{33}$ Briefly, a mixed solution of phospholipids in chloroform was dried and formed a lipid film in a round-bottom flask. The lipid film was then hydrated in a hydration solution containing 150 mM DA or 5-HT,

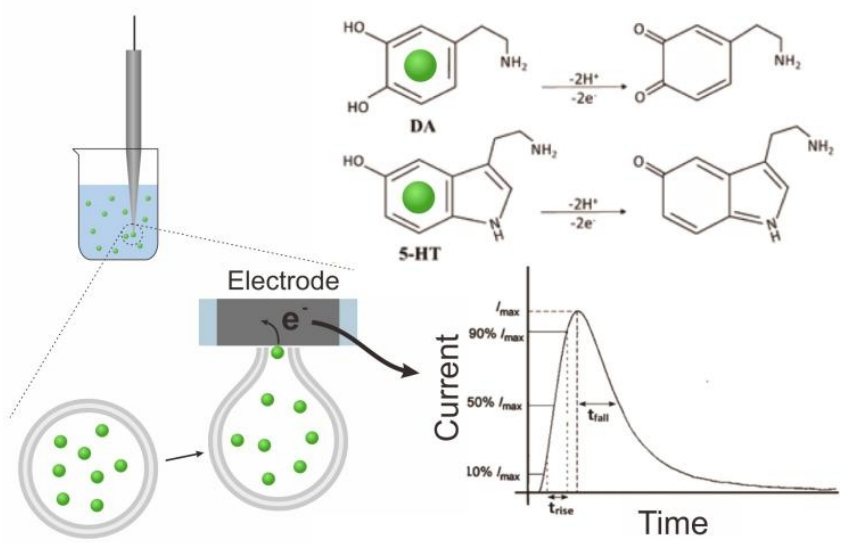

Fig.1 Schematic showing the VIEC principle: attachment of the liposomes onto an electrode, electrooxidation of the released monoamines, and convection to a typical amperometric spike for peak analysis. The inset shows the electrooxidation of DA and 5-HT.

To prepare a population of DLL or SLL with various sizes. The sizes of vesicles were narrowed by use of reciprocating extrusion through double polycarbonate filter membranes in the same hydration solution and were measured to be $188 \mathrm{~nm}$ (polydispersity index $=0.13$ ) as the average radius via dynamic light scattering (see more details in Supporting Information).

The DLL or SLL are stable in isotonic solution. To trigger and record the release of contents, VIEC was used according as described previously ${ }^{33}$ (see Figure 1, and more details in Supporting Information). When a vesicle settles on the electrode, the high electric field near the surface induces electroporation of the liposome membrane allowing the contents to diffuse out through the pore in membrane at the electrode surface. Released DA and 5-HT are rapidly electrooxidized at the electrode generating a spike shaped current. Using Faraday's law, the charge passed for each current spike $(Q$, the charge) is proportional to the number of molecules oxidised. In addition, the dynamic parameters of each spike, including the rise time $\left(t_{\text {rise }}\right)$, fall time $\left(t_{\text {fall }}\right)$, and width at half spike height $\left(t_{\text {half }}\right)$ can be used to evaluate release dynamics. ${ }^{17}$

\section{Comparison of the release dynamics of DLL and SLL}

By applying the VIEC approach to each group of DLL and SLL, the dynamics of release from the vesicles in each group were statistically analysed (2182 spikes for DLL and 2301 spikes for SLL, 10 traces for each, see details in Supporting Information). Typical amperometry traces for DLL and SLL and the spike analysis parameters are shown in Figure 2 and Figure 3, respectively. The results reveal that the average quantities of both monoamine molecules within each individual liposome and the height of the spike $\left(I_{\max }\right)$ of both groups are similar, 
while a significant increase in $t_{\text {half }}$ and also a relative decrease in fall time $\left(t_{\text {fall }}\right)$ is observed in the SLL compared to the DLL. Typical spikes (in the form of logarithmic dimensionless current) for each of DLL and SLL are shown to the right of Figure 2. The decay of the DLL spike has been fit to a rapid decay ( $1 \mathrm{~ms})$ and subsequent slow long decay ( $\sim 10 \mathrm{~ms})$, and it is better fit to a DblExp decay function than a SigExp function, whereas the spike of SLL fits better to only a a SigExp function. We further

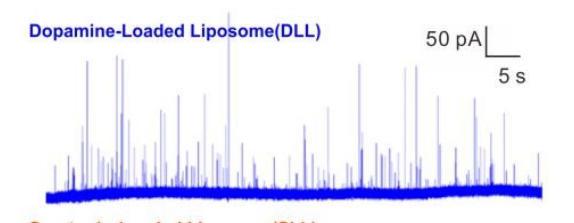

Serotonin-Loaded Liposome(SLL)
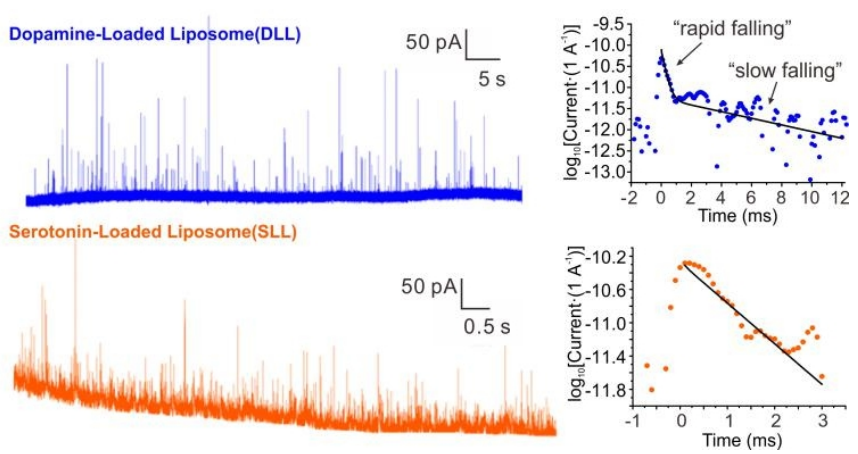

Fig.2 Traces for VIEC of DLL (blue line) and SLL (orange line) with a typical spike for each blown up to the right (with $\log _{10} Y$ axis, blue dots for DLL, orange dots for SLL), showing the difference in spikes shape. The best-fit simulation results (see the The simulated result for DLL follows a DbIExp while that for the SLL is a SigExp.

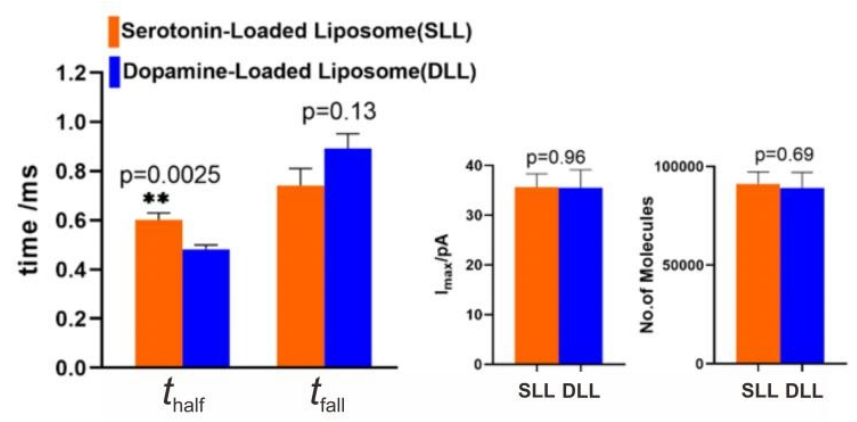

Fig.3 Comparison of DLL vs. SLL VIEC data showing means for dynamic data: $t_{\text {half }}$ $t_{\text {fall }}, I_{\max }$, and number of molecules observed in each. The fall time is defined as 90 a two-tailed Mann-Whitney rank-sum test; ${ }^{* *}, \mathrm{p}<0.01$.

compared the number of spikes having DbIExp vs. SigExp decay for all spikes from DLL and SLL. Indeed, when the ratio of $\chi^{2}$ (single) $/ \chi^{2}$ (double) $>1$ set as a cutoff, the percentage of spikes that have a better fit to DbIExp decay in the DLL group ( 70\%) is higher than for the SLL group ( 60\%). This result shows that dopamine release from the liposomes is more complex and generally slower than 5-HT, which might result from the slower desorption of DA from membrane lipids (liposomes have no dense core).

\section{The desorption-release processes of DA and 5-HT from liposomes}

Theoretically, as discussed previously, ${ }^{17,22,37}$ the dynamics of release from liposomes (or vesicles) is thought to be mainly controlled by the product of diffusional flux and the pore area. In contrast to exocytosis where pore opening and closing is driven by the cell, the liposome pore cannot close under a constant strong electric field. ${ }^{38}$ Thus, the outflow flux should fit to a SigExp decay following the diffusion model of Cottrell. ${ }^{22}$ But if a rate-limiting desorption process is added before release through the pore, the entire release rate becomes a combination of desorption rate and diffusion (via pore) rate, and both can be fit to SigExp decay with different scaling factors.
If the desorption rate is faster, the release rate will be controlled only by the slower diffusion rate and fit to a single exponential decay (similar to the apparent rate of a multi-step reaction controlled by the rate-determining step). This is shown conceptually in Figure 4. However, when the rates of the two processes are similar, the apparent rate of the combined process will be expressed as a DbIExp mode.

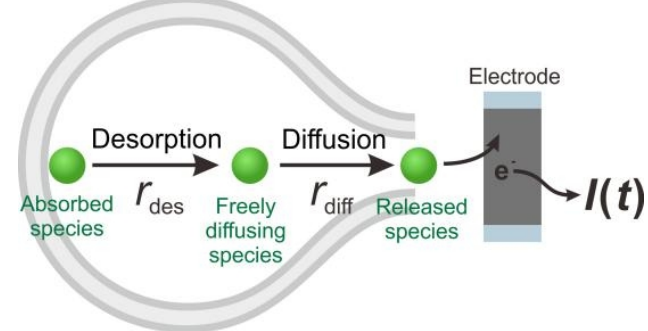

Fig.4 A schematic of the desorption-release process. The adsorbed species dissociates from the lipid membrane creating a freely diffusing species. These species are free to exit via the pore as released species. This mechanism is analogous to $a 1^{\text {st }}$ order chemical reaction or one that is further combined as two consecutive reactions and the rate limiting step determines the apparent rate and whether the fall tie is a single of double exponential. The $r_{\text {des }}$ is the desorption rate of absorbed species. The $r_{\text {diff }}$ is the diffusion rate (i.e. flux) of freely diffusing species.

We constructed a finite element model to simulate this desorption-release process. The finite element model depicts a spherical liposome (radius $188 \mathrm{~nm}$, experimental average) with a round pore in its membrane. On the other side, the total quantity of chemical cargo was set as 151000 molecules (according to the average experimental current spikes of charge $Q=48 \mathrm{fC}$ ), but divided into absorbed species $\left(Q_{\mathrm{s}}\right.$, where $\mathrm{s}$ indicates the membrane inner surface) and freely diffusing species $\left(Q_{\mathrm{f}}\right.$, findicates the freely moving species). Species need to reach an adsorption-desorption equilibrium before release. If we pre-set parameters describing the adsorption-desorption process, such as the adsorption rate constant $\left(k_{\text {ads }}\right)$, of adsorbed molecules in the saturated state $\left(\Gamma_{\mathrm{s}}\right)$, then the $Q_{\mathrm{s}}$ and $Q_{\mathrm{f}}$ can be calculated by use of Langmuir's adsorption equation and converted into an initial concentration on the membrane $\left(C_{\mathrm{s}, 0}\right)$ and in the liposome cavity $\left(C_{\mathrm{f}, 0}\right)$. After pore generation, the freely diffusing species exit the pore, while the absorbed species require time to dissociate from the membrane and transfer into the solution phase. The flux of molecules flowing out the pore is converted into current that is then used to fit to the experimental spike and estimate the key parameters in the desorption-release process, including the desorption rate constant $\left(k_{\mathrm{des}}\right)$ and radius of the pore $\left(R_{\mathrm{p}}\right)$. The Monte Carlo optimization method was used to facilitate these estimates owing to its advantages such as high efficiency to solve complex problems and exemption of any a priori hypothetical relationship between current spikes and initial parameters (see calculation protocol and details in Supporting Information).

Four groups of typical DA and 5-HT spikes were analyzed by the finite element simulation. A pair of the experimental spikes and their best fit results are shown in Figure 2. The DLL spikes show a better fit with a DblExp decay, whereas the SLL spikes fit better to a SigExp decay. By comparing the estimated desorption rate constant $\left(k_{\text {des }}\right)$ and pore radius $\left(R_{\mathrm{p}}\right)$ corresponding to DA or $5-\mathrm{HT}$ spikes, the pore sizes of both groups (see Table 1 ) cover a similar range (40 90 nm for DLL vs. $40 \sim 80 \mathrm{~nm}$ for SLL), while the $k_{\mathrm{des}}$ of 
DA is obviously smaller than $5-\mathrm{HT}\left(10^{2.3^{\sim 3.0}} \mathrm{~s}^{-1}\right.$ for DA vs. $10^{3.5^{\sim 5}}$.1 $\mathrm{s}^{-1}$ for $5-\mathrm{HT}$ ). This smaller $k_{\text {des }}$ leads to the slower desorption rate $\left(r_{\text {des }}\right)$ and longer time to end of the release, which is consistent with a longer decay for DLL spikes. The smaller $k_{\text {des }}$ of DA may result from the greater number of $\mathrm{H}$-bonds ( 3 bonds) formed Table 1. The estimated results of 4 pairs of spikes from DLL and SLL

\begin{tabular}{ccccc} 
& \multicolumn{2}{c}{ DLL } & \multicolumn{2}{c}{ SLL } \\
\cline { 2 - 5 } No. & $\begin{array}{c}\log _{10} k_{\mathrm{des}} \\
(1 / \mathrm{s})\end{array}$ & $\begin{array}{c}R_{\mathrm{p}} \\
(\mathrm{nm})\end{array}$ & $\begin{array}{c}\log _{10} k_{\mathrm{des}} \\
(1 / \mathrm{s})\end{array}$ & $\begin{array}{c}R_{\mathrm{p}} \\
(\mathrm{nm})\end{array}$ \\
\hline 1 & 2.31 & 43 & 3.93 & 43 \\
\hline 2 & 3.05 & 64 & 3.48 & 56 \\
\hline 3 & 2.77 & 93 & 4.43 & 47 \\
\hline 4 & 2.90 & 74 & 5.12 & 80 \\
\hline
\end{tabular}

between dopamine and the polar neutral lipid compared to serotonin which can form only 2 . The higher free energy of DA to lipid binding $(\sim 21 \mathrm{~kJ} / \mathrm{mol})$ compared to $5-\mathrm{HT}(\sim 14 \mathrm{~kJ} / \mathrm{mol})^{27}$ make it harder for DA to dissociate.

These results support our assumption that different desorption rates alter the release dynamics between these two monoamines (Figure 5). It also provides a new perspective for the DblExp decay in the study of bio-vesicular release by the VIEC experiment or exocytosis measured by single cell amperometry. However, it is worth noting that, in the VIEC analysis of native biological vesicles, the proportion of DblExp fitting (empirically $40 \% \sim 60 \%)^{39}$ is much higher than that of liposomes (empirically $<10 \%$, when the ratio of $\chi^{2}$ (single) $/ \chi^{2}$ (double) $>2$ set as the cutoff). This phenomenon likely indicates that the dense core in the bio-vesicles still also plays an important role in regulating release from the vesicle. ${ }^{18,40-43}$

\section{Conclusions}

In summary, we employed the VIEC technique to monitor the differential dynamics of chemical cargo release from liposomes loaded with two different electroactive neurotransmitters (DA and $5-\mathrm{HT}$ ). Liposomes were used to simulate bio-vesicles without a dense core or proteins. A finite element simulation with Monte Carlo optimization was adopted to estimate the kinetic parameters of chemical desorption and suggests that the rate constant of DA desorbing from the lipids inside the vesicle Is smaller than that for $5-\mathrm{HT}$. The slower desorption alters the release rate as recognized by a mode of best fit (SigExp or DblExp) for spike signal decay. Our models suggest this might be induced by a stronger interaction between cargo and vesicle membrane lipids, as more hydrogen bonding between DA and membrane lipid molecules can occur than for 5-HT. Hence, the existence of adsorption-desorption behaviour for transmitter molecules and the lipid membrane could be a basic component of the vesicular release process. Although the dense core might also play a crucial role in regulating chemical release from the cells having vesicles containing protein dense cores, regulation via adsorption-desorption from the vesicle wall can occur in all biological vesicles with or without a dense core. A better understanding of the interaction between transmitters and the vesicle membrane might provide strategies to regulate neurotransmitter, hormone, protein or drug release related to cellular communication, intracellular vesicular transport, and controlled delivery of liposomal drugs.

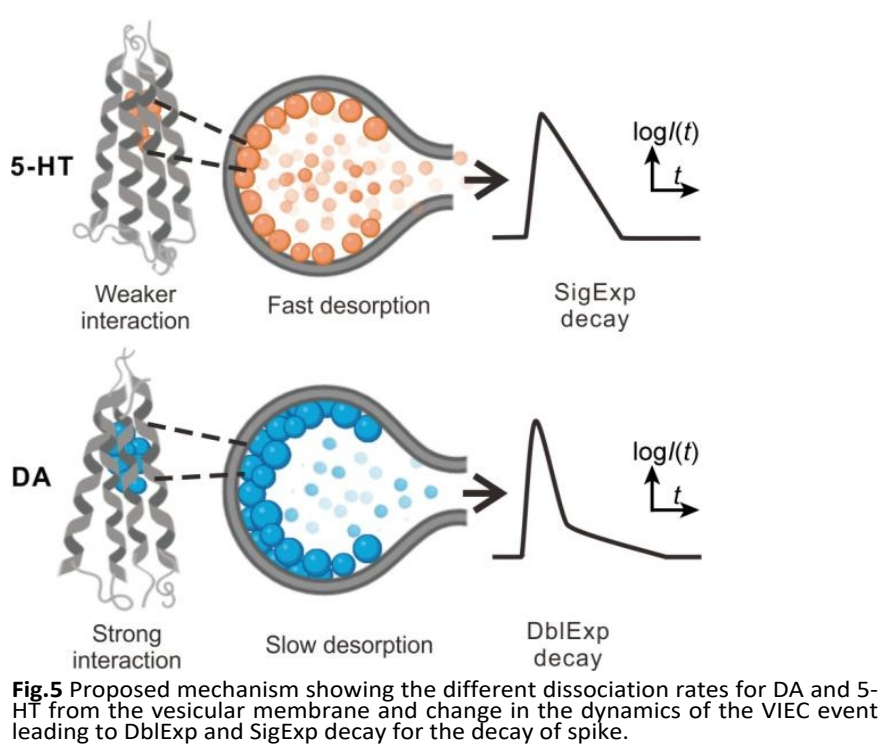

\section{Author Contributions}

F. Asadpour and X. Zhang contributed equally. All authors have given approval to the final version of the manuscript.

\section{Conflicts of interest}

The authors declare no competing financial interest.

\section{Acknowledgements}

The European Research Council (ERC Advanced Grant Project No 787534 NanoBioNext), Knut and Alice Wallenberg Foundation, and the Swedish Research Council (VR Grant No 2017-04366) are acknowledged for financial support. FA, MMA, and MA wish to thank the Yazd University Research Council and the Iran National Science Foundation (INSF) for financial support of this research.

\section{References}

1 R. Jahn and T. C. Südhof, Annu. Rev. Biochem., 1999, 68, 863911.

2 R. C. Lin and R. H. Scheller, Annu. Rev. Cell Dev. Bi., 2000, 16, 19-49.

3 M. Vakilian, Y. Tahamtani and K. Ghaedi, Gene, 2019, 706, 5261.

4 N. T. N. Phan, X. Li and A. G. Ewing, Nat. Rev. Chem., 2017, 1.

5 R. S. Zucker, Neuron, 1996, 17, 1049-1055.

6 R. D. Burgoyne and A. Morgan, Physiol. Rev., 2003, 83, 581632.

7 C. Amatore, S. Arbault, M. Guille and F. Lemaitre, Chem. Rev., 2008, 108, 2585-2621. 
8 W. Wang, S. H. Zhang, L. M. Li, Z. L. Wang, J. K. Cheng and W. H. Huang, Anal. Bioanal. Chem., 2009, 394, 17-32.

9 R. M. Wightman, J. A. Jankowski, R. T. Kennedy, K. T. Kawagoe, T. J. Schroeder, D. J. Leszczyszyn, J. A. Near, E. J. Diliberto and O. H. Viveros, Proc. Natl. Acad. Sci. U. S. A., 1991, 88, 1075410758.

10 A. Schulte and W. Schuhmann, Angew. Chem. Int. Ed., 2007, 46, 8760-8777.

11 P. Sun, F. O. Laforge, T. P. Abeyweera, S. A. Rotenberg, J. Carpino and M. V. Mirkin, Proc. Natl. Acad. Sci. U. S. A., 2008, 105, 443-448.

12 H. K. McCormick and J. E. Dick, Anal. Bioanal. Chem., 2021, 413, 17-24.

13 R. Jahn, T. Lang and T. C. Südhof, Cell, 2003, 112, 519-533.

14 T. C. Sudhof and J. E. Rothman, Science, 2009, 323, 474-477.

15 J. Dunevall, H. Fathali, N. Najafinobar, J. Lovric, J. Wigstr M, A. Cans and A. G. Ewing, J. Am. Chem. Soc., 2015, 137, 43444346.

16 J. Dunevall, S. Majdi, A. Larsson and A. Ewing, Curr. Opin. Electrochem., 2017, 5, 85-91.

17 E. V. Mosharov and D. Sulzer, Nat. Methods, 2005, 2, 651-658.

18 A. Oleinick, R. Hu, B. Ren, Z. Tian, I. Svir and C. Amatore, J. Electrochem. Soc., 2016, 163, H3014-H3024.

19 R. Trouillon and A. G. Ewing, ChemPhysChem, 2013, 14, 22952301.

20 R. Trouillon and A. G. Ewing, ACS Chem. Biol., 2014, 9, 812820.

21 X. Li, A. S. Mohammadi and A. G. Ewing, J. Electroanal. Chem., 2016, 781, 30-35.

22 C. Amatore, A. I. Oleinick and I. Svir, ChemPhysChem, 2010, 11, 149-158.

23 Q. Yue, X. Li, F. Wu, W. Ji, Y. Zhang, P. Yu, M. Zhang, W. Ma, M. Wang and L. Mao, Angew. Chem. Int. Ed., 2020, 59, 1106111065.

24 R. M. Wightman, K. P. Troyer, M. L. Mundorf and R. Catahan, Ann. Ny. Acad. Sci., 2002, 971, 620-626.

25 K. Jodko-Piorecka and G. Litwinienko, ACS Chem. Neurosci., 2013, 4, 1114-1122.

26 G. H. Peters, C. Wang, N. Cruys-Bagger, G. F. Velardez, J. J. Madsen and P. Westh, J. Am. Chem. Soc., 2013, 135, 21642171.

27 P. A. Postila, I. Vattulainen and T. Róg, Sci. Rep., 2016, 6, 19345.

28 A. Datta, C. L. Haynes and V. H. Barocas, Integr. Biol., 2017, 9, 248-256.

29 C. Gu, X. Zhang and A. G. Ewing, Anal. Chem., 2020, 92, 1026810273.

30 R. Trouillon, Y. Lin, L. J. Mellander, J. D. Keighron and A. G. Ewing, Anal. Chem., 2013, 85, 6421-6428.

31 W. Cheng and R. G. Compton, Angew. Chem. Int. Ed., 2014, 53 13928-13930.

32 W. Cheng and R. G. Compton, ChemElectroChem, 2016, 3, 2017-2020.

33 J. Lovric, N. Najafinobar, J. Dunevall, S. Majdi, I. Svir, A. Oleinick, C. Amatore and A. G. Ewing, Faraday Discuss., 2016 193, 65-79.

34 A. S. Cans, N. Wittenberg, R. Karlsson, L. Sombers, M. Karlsson, O. Orwar and A. Ewing, Proc. Natl. Acad. Sci. U. S. A. 2003, 100, 400-404.

35 C. Wang, F. Ye, G. F. Velardez, G. H. Peters, P. Westh and T. P. Abeyweera, J. Phys. Chem. B, 2011, 115, 196-203.

36 G. Taugner and A. Wahler, Naunyn-Schmiedeberg's Arch. Pharmacol., 1974, 282, 279-293.

37 C. Amatore, A. I. Oleinick and I. Svir, ChemPhysChem, 2010, 11, 159-174.

38 A. O. Bilska, K. A. DeBruin and W. Krassowska, Bioelectrochemistry, 2000, 51, 133-143.
39 X. W. Zhang, A. Hatamie and A. G. Ewing, J. Am. Chem. Soc., 2020, 142, 4093-4097.

40 J. D. Machado, J. Diaz-Vera, N. Dominguez, C. M. Alvarez, M. R. Pardo and R. Borges, Cell. Mol. Neurobiol., 2010, 30, 11811187.

41 R. Borges, J. Díaz Vera, N. Domínguez, M. R. Arnau and J. D. Machado, J. Neurochem., 2010, 114, 335-343.

42 R. Hu, B. Ren, C. Lin, A. Oleinick, I. Svir, Z. Tian and C. Amatore, J. Electrochem. Soc., 2016, 163, H853-H865.

$43 \mathrm{~N}$. Dominguez, J. Estevez-Herrera, R. Borges and J. D. Machado, FASEB J., 2014, 28, 4657-4667. 


\section{SUPPORTING INFORMATION}

\section{Vesicular Release Dynamics are Altered by Interaction between Chemical Cargo and Vesicle Membrane Lipids}

Farzaneh Asadpour, ${ }^{\ddagger a b}$ Xinwei Zhang, ${ }^{\ddagger a}$ Mohammad Mazloum-Ardakani, ${ }^{* b}$ Meysam Mirzaei, ${ }^{\mathrm{c}}$ Soodabeh Majdib, ${ }^{a}$ Andrew G. Ewing ${ }^{* a}$

a) Department of Chemistry and Molecular Biology, University of Gothenburg, 41296 Gothenburg, Sweden.*E-mail: Andrew.ewing@chem.gu.se

b) Department of Chemistry, Faculty of Science, Yazd University, Yazd, 89195-741, Iran. *E-mail: Mazloum@yazd.ac.ir

c) Department of Materials Science and Engineering, School of Engineering, Shiraz University, Shiraz, Iran.

\section{Table of Contents:}

\section{Experimental Details}

Chemicals

Solutions

Liposomes preparation

VIEC experiments

\section{Numerical Simulation Analysis}

The building of a finite element model

Figure S1. The schematics of the model configuration at initial time and afterward Table S1. Model input parameters

Inverse estimation of desorption parameters based on Monte Carlo method 


\section{EXPERIMENTAL DETAILS}

\section{Chemicals}

1,2-dioleoyl-sn-glycero-3-phosphocholine (DOPC, >99\%), 1,2-dioleoyl-sn-glycero-3phosphethanolamine (DOPE, $>99 \%$ ) and cholesterol (ovine wool, $>98 \%$ ) were purchased from Avanti Polar Lipids, USA. Serotonin hydrochloride $(\geq 98.0 \%)$, dopamine hydrochloride $(98.0 \%)$, chloroform ( $\geq 99.9 \%)$, methanol $(\geq 99.9 \%)$ were obtained from Sigma-Aldrich. All aqueous solutions were prepared using $18 \mathrm{M} \Omega \cdot \mathrm{cm}^{-1}$ water from Purelab Classic purification system (ELGA, Sweden).

\section{Solutions}

Lipid film hydration buffer for preparing liposomes:

The lipid film hydration was performed in a solution of $150 \mathrm{mM}$ dopamine (DA)/serotonin (5-HT) hydrochloride in $10 \mathrm{mM}$ HEPES at $\mathrm{pH}$ 7.4.

Pre-equilibration buffers for the Sephadex G-25 column:

The Sephadex G-25 column for removing free DA/5-HT outside liposomes was preequilibrated with a buffer containing $142 \mathrm{mM} \mathrm{NaCl}$ in $10 \mathrm{mM}$ HEPES with $\mathrm{pH}$ 7.4.

Liposome isotonic buffer:

The storage, amperometric measurements and nanoparticle tracking analysis (NTA) of liposome samples were performed in buffer with $10 \mathrm{mM}$ HEPES, $142 \mathrm{mM} \mathrm{NaCl}, \mathrm{pH}$ 7.

All buffers were purged with argon for 30 min before adding oxygen sensitive DA/5HT hydrochloride.

\section{Liposome preparation}

Neurotransmitter-loaded liposomes were prepared passively by thin lipid film hydration. A solution of 1,2-dioleoyl-sn-glycero-3- phosphocholine (DOPC), 1,2dioleoyl-sn-glycero-3-phosphethanolamine (DOPE) and cholesterol (60:20:20 mole ratio) in chloroform was dried in a round-bottom flask by rotary evaporation until a lipid film was obtained $(\sim 3 \mathrm{~h})$. The dried lipid cake was re-hydrated to obtain a liposome suspension by gently mixing with $1.5 \mathrm{~mL}$ of the hydration buffer and then left to stand for $30 \mathrm{~min}$ under argon gas at room temperature. The liposome suspension was freeze-thawed in liquid nitrogen 3 to 5 times to form multilamellar vesicles, and then extruded 11 times through double polycarbonate membranes of $0.4 \mu \mathrm{m}$ pore size with an Avanti Mini-Extruder (Avanti Polar Lipids, Inc., USA). This procedure yielded liposomes with a mean diameter of $376 \mathrm{~nm}$, as measured by dynamic light scattering (DLS) on a Malvern Zetasizer Nano ZS (Malvern Inc., Malvern, UK). Removal of free DA or 5-HT outside liposomes was achieved by gel filtration on a Sephadex G-25 column which was pre-equilibrated in advance. 


\section{VIEC experiments}

Monitoring the cargo released was accomplished by vesicle impact electrochemical cytometry to detect DA or 5-HT stored in liposomes. A 33- $\mu$ m carbon fiber electrode was placed in a liposome suspension as shown in Figure 1 of the main text. Liposomes adsorbed on the microelectrode and ruptured via electroporation, the proposed mechanism for vesicle opening in the VIEC. The electrochemical recording of release of individual liposome content was performed by applying a constant positive potential ( $700 \mathrm{mV} v s . \mathrm{Ag} / \mathrm{AgCl}$ ) to the carbon fiber microelectrode in the form of spikes (a plot of current versus time). By analysis of each spike, important kinetic information about the release event can be obtained. Several parameters used for spike analysis in this work are depicted in the schematic in Figure 1 of the main text including the width of spike at half maximum ( $\left.t_{\text {half }}\right)$ as an indicator of duration of release events; the rise-time $\left(t_{\text {rise }}\right)$, the time from $10 \%$ to $90 \%$ of amplitude on the rising part of each spike, which reflects fusion pore opening; the fall-time $\left(t_{\text {fall }}\right)$, the time from $90 \%$ to $10 \%$ of amplitude on the falling part of each spike that represents the time needed for chemical contents to exit the liposome, and the amplitude of spike $\left(I_{\max }\right)$ representing the maximum flux of molecules through the open pore. Additionally, the amount of monoamines $(N)$ released from a single liposome was evaluated with Faraday's law: $N=Q / z_{\mathrm{e}} \mathrm{F}$, where $Q$ is the charge calculated by integrating current of each amperometric spike, $z_{\mathrm{e}}$ is the number of electrons transferred during the redox reaction ( 2 for DA or 5-HT) and F is Faraday's constant $\left(96485 \mathrm{C} \cdot \mathrm{mol}^{-1}\right)$.

\section{Carbon fiber microelectrode (CFE) fabrication}

Vesicle impact electrochemical cytometry (VIEC) measurements were performed using amperometry at a carbon fiber microelectrode placed in a liposome suspension. The microelectrodes were prepared by aspirating a 33- $\mu \mathrm{m}$ carbon fiber into a glass capillary (o.d. $1.2 \mathrm{~mm}$; i.d. $0.69 \mathrm{~mm}$; no filament; Sutter Instrument Co., USA). A commercial micropipette puller (PE-21, Narishige, Japan) was used to heat and pull the capillary producing two carbon fiber-containing pipettes. A scalpel was used to cut the protruding carbon fiber close to the glass tip and it was then dipped into freshly made epoxy (EpoTek 301, Epoxy Technology, USA) for $3 \mathrm{~min}$. The glued electrodes were cured at $100{ }^{\circ} \mathrm{C}$ overnight and subsequently cut at the glass junction. The electrodes were consequently polished at a $45^{\circ}$ angle on a commercial micro grinder (EG-400, Narishige, Japan) and backfilled with $3 \mathrm{M} \mathrm{KCl}$. Each electrode was then tested in a 0.1 $\mathrm{mM}$ solution of dopamine in phosphate-buffered saline (PBS; $\mathrm{pH}$ 7.4) by performing cyclic voltammetry. Only electrodes with proper $I-E$ curves were used for experiments.

\section{Amperometric measurements}

The electrode was kept at $700 \mathrm{mV}$ vs. a $\mathrm{Ag} / \mathrm{AgCl}$ reference/counter electrode (World Precision Instruments, Inc., USA) using an amplifier instrument (Axopatch 200B, Axon Instruments, USA). The signal was digitized at $10 \mathrm{kHz}$ and filtered with an internal low pass Bessel filter at $2 \mathrm{kHz}$. The signal was displayed in real time (AxoScope 8.1, Axon Instruments, USA) and stored digitally.

Data Acquisition and Statistical analysis 
The amperometric traces were processed by a macro of Igor Pro 6 (Version 6.3.7.2; Page 16 of 24 WaveMetrics, Lake Oswego, OR) designed for analysis of quantal release by the group of David Sulzer at Columbia University. Peaks were detected if they exceeded a threshold of 3 times the RMS noise. Statistical analysis for amperometric measurements data were performed in GraphPad Prism 7 (GraphPad Software, Inc., San Diego, CA). The average of all parameters are calculated as the mean of medians of all recorded traces. 


\section{NUMERICAL SIMULATION ANALYSIS}

\section{The building of the finite elemental model}

The finite elemental model for liposomal release was based on the model of a disk electrode detecting exocytosis in our previous work ${ }^{1}$, with a new module for calculating the adsorption-desorption dynamics was added (scheme in Figure S1).
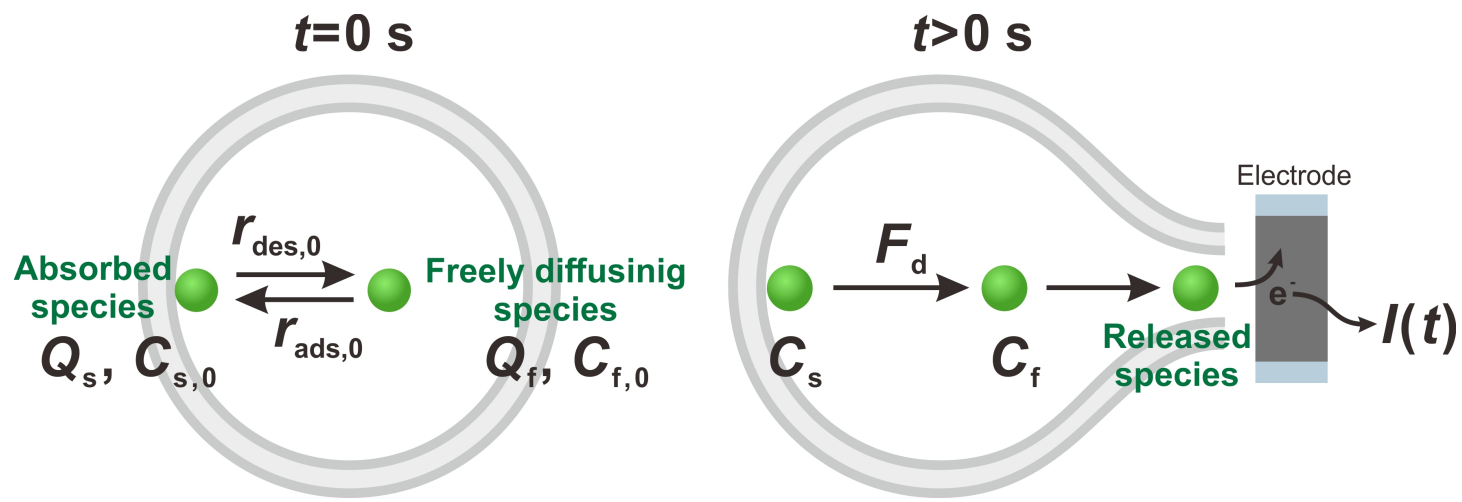

Figure S1. Schematic of the model configuration at initial time $(\mathrm{t}=0 \mathrm{~s})$ and after instantaneous electroporation ( $\mathrm{t}>0 \mathrm{~s}$ ).

Schematically, a circle (the radius, $R_{\text {lip }}=188 \mathrm{~nm}$ ) and a pore on the membrane with variable size $\left(R_{\mathrm{p}}\right)$ was used to simulate the electroporated liposome membrane where a total quantity of cargo ( $Q=48.4 \mathrm{fC}$, the average experimental charge for spikes from DA/5-HT groups) was initially set inside the vesicles, but separated into adsorbed spcies $\left(Q_{\mathrm{s}}, \mathrm{s}\right.$ indicates the membrane inner surface) and free species $\left(Q_{\mathrm{f}}\right.$, $\mathrm{f}$ indicates freely moving species). The values for $Q_{\mathrm{s}}$ and $Q_{\mathrm{f}}$ were calculated a priori based on the Langmuir adsorption model.

At time $=0 \mathrm{~s}$, the initial surface concentration $\left(C_{\mathrm{s}, 0}\right)$ and initial concentration of intraliposomal freely moving molecules $\left(C_{\mathrm{f}, 0}\right)$ was assumed to be at equilibrium, so the initial adsorption rate $\left(r_{\mathrm{ads}, 0}\right)$ was equal to the desorption rate $\left(r_{\mathrm{des}, 0}\right)$.

$$
r_{\mathrm{des}, 0}=k_{\mathrm{des}} C_{\mathrm{s}, 0}=r_{\mathrm{ads}, 0}=k_{\mathrm{ads}} C_{\mathrm{f}, 0}\left(\Gamma_{\mathrm{s}}-C_{\mathrm{s}, 0}\right)
$$

Where $k_{\text {ads }}$ is the adsorption rate constant, $k_{\text {des }}$ is the desorption rate constant, $\Gamma_{\mathrm{s}}$ is the surface concentration of adsorbed molecules in saturated state on the membrane. Hence, the initial surface concentration $\left(C_{\mathrm{s}, 0}\right)$ can be calculated by

$$
C_{\mathrm{s}, 0}=\frac{k_{\mathrm{ads}} C_{\mathrm{f}, 0} \Gamma_{\mathrm{s}}}{k_{\mathrm{des}}+k_{\mathrm{ads}} C_{\mathrm{f}, 0}}
$$

Meanwhile, the total spike charge $Q=Q_{\mathrm{s}}+Q_{\mathrm{f}}$, and

$$
Q_{\mathrm{s}}=2 \mathrm{~F}\left(4 \pi R_{\mathrm{lip}}^{2}\right) C_{\mathrm{s}, 0} ; Q_{\mathrm{f}}=2 \mathrm{~F}\left(4 \pi R_{\mathrm{lip}}^{3} / 3\right) C_{\mathrm{f}, 0}
$$

where $\mathrm{F}$ is the Faraday constant. Then $C_{\mathrm{f}, 0}$ was solved by equation 1 ,

$$
\frac{1}{3} R_{\mathrm{lip}} C_{\mathrm{f}, 0}{ }^{2}+\left(\Gamma_{\mathrm{s}}+\frac{1}{3} R_{\mathrm{lip}} K_{1}-Q / 8 \mathrm{~F} \pi R_{\mathrm{lip}}{ }^{2}\right) C_{\mathrm{f}, 0}-Q K_{1} / 8 \mathrm{~F} \pi R_{\mathrm{lip}}{ }^{2}=0
$$

where $K_{1}$ is the Langmuir constant, which equals $k_{\text {des }} / k_{\text {ads. }}$. 
After the initial state was set, a flux inward, from the circle, simulating the desorption, was determined by $F_{\text {d, }}$

$$
F_{\mathrm{d}}=k_{\mathrm{ads}} C_{\mathrm{f}}\left(\Gamma_{\mathrm{s}}-C_{\mathrm{s}}\right)-k_{\mathrm{des}} C_{\mathrm{s}}
$$

Notably, the value of $k_{\mathrm{ads}} C_{\mathrm{f}}\left(\Gamma_{\mathrm{s}}-C_{\mathrm{s}}\right)$ is usually much less than $k_{\mathrm{des}} C_{\mathrm{s}}$, so $F_{\mathrm{d}} \approx-k_{\mathrm{des}} C_{\mathrm{s}}$, which results in the influence of $k_{\text {ads }}$ and $\Gamma_{\mathrm{s}}$ is small and, nence, their estimation is quite unreliable.

When $\mathrm{t}>0 \mathrm{~s}$, the real time concentrations of surface absorption molecules $\left(C_{\mathrm{s}}\right)$ and freely moving molecules $\left(C_{\mathrm{f}}\right)$ were solved by use of Fick's diffusion equation with a flux inward.

$$
\begin{aligned}
& \frac{\partial C_{\mathrm{s}}}{\partial t}+\nabla \cdot\left(-D_{\mathrm{s}} \nabla C_{\mathrm{s}}\right)=-F_{\mathrm{d}} \\
& \frac{\partial C_{\mathrm{f}}}{\partial t}+\nabla \cdot\left(-D_{\mathrm{f}} \nabla C_{\mathrm{f}}\right)=F_{\mathrm{d}}
\end{aligned}
$$

Here, $\mathrm{t}$ is the time, $D_{\mathrm{s}}$ and $D_{\mathrm{f}}$ is the diffusion coefficient of surface molecules and freely moving molecules. The input parameters are listed in Table S1.

Finally, a current reflecting the release dynamics was solved by use of Faraday's law and surface integration of the flux density of molecules across the pore,

$$
I(t)=\oiint_{S \text { pore }} z \mathrm{e} F J(t) \mathrm{d} S
$$

where $J(t)$ is the flux density of molecules across the liposome pore, $z_{\mathrm{e}}$ is the charge transfer number of the electrochemical reaction which is 2, and F is the Faraday constant.

Table S1. Model input parameters

\begin{tabular}{cccc} 
Parameters & Description & Value & Unit \\
\hline$D_{\mathrm{f}}$ & diffusion coefficient of chemicals & $6.0 \times 10^{-10}($ Ref. 2) & $\mathrm{m}^{2} \cdot \mathrm{s}^{-1}$ \\
\hline$D_{\mathrm{s}}$ & $\begin{array}{c}\text { surface diffusion coefficient of } \\
\text { chemicals }\end{array}$ & 0 & $\mathrm{~m}^{2} \cdot \mathrm{s}^{-1}$ \\
\hline$C_{\mathrm{f}, 0}$ & $\begin{array}{c}\text { initial concentration of free } \\
\text { chemical inside liposome }\end{array}$ & solution of Eq.1 & $\mathrm{mol} / \mathrm{m}^{3}$ \\
\hline$Z_{\mathrm{e}}$ & $\begin{array}{c}\text { number of electrons transferred in } \\
\text { the electrochemical reaction }\end{array}$ & 2 & \\
\hline$R_{\text {lip }}$ & the radius of liposome & 188 & $\mathrm{~nm}$ \\
\hline$Q$ & $\begin{array}{c}\text { the total charge according to } \\
\text { chemical in a liposome }\end{array}$ & $4.84 \times 10^{-14}$ & $\mathrm{C}$ \\
\hline
\end{tabular}

\section{Inverse estimation of desorption parameters based on the Monte Carlo method}

The inverse estimation was based on a Monte Carlo least-squares optimization method. The model parameters include $k_{\mathrm{des}}, k_{\mathrm{ads}}, \Gamma_{\mathrm{s}}, R_{\mathrm{p}}$. The experimental spike signal was set as the target, and the least sum of the squared difference between the target and the corresponding value calculated by the above finite elemental model was used to search 
the best-fitting combination of desired parameters. As discussed above, the estimation of $k_{\mathrm{ads}}$ and $\Gamma_{\mathrm{s}}$ is unreliable, so only the estimated $k_{\mathrm{des}}$ and $R_{\mathrm{p}}$ of the typical spikes are listed in Table 1.

\section{References}

(1) Gu, C.; Zhang, X.; Ewing, A. G. Comparison of Disk and Nanotip Electrodes for Measurement of Single-Cell Amperometry during Exocytotic Release. Anal. Chem. 2020, 92, 10268-10273.

(2) Trouillon, R.; Lin, Y.; Mellander, L. J.; Keighron, J. D.; Ewing, A. G. Evaluating the Diffusion Coefficient of Dopamine at the Cell Surface During Amperometric Detection: Disk Vs Ring Microelectrodes. Anal. Chem. 2013, 85, 6421-6428. 